\title{
UNIVERSITY OF WISCONSIN
}

\section{Center for Plasma Theory and Computation REPORT}

Poloidal Variation of Viscous Forces in the Banana Collisionality Regime

\author{
J.P. Wang and J.D. Callen \\ Nuclear Engineering \& Engineering Physics \\ and Physics Departments \\ University of Wisconsin-Madison, \\ Madison, Wisconsin 53706
}

December 1992

UW-CPTC 92-6

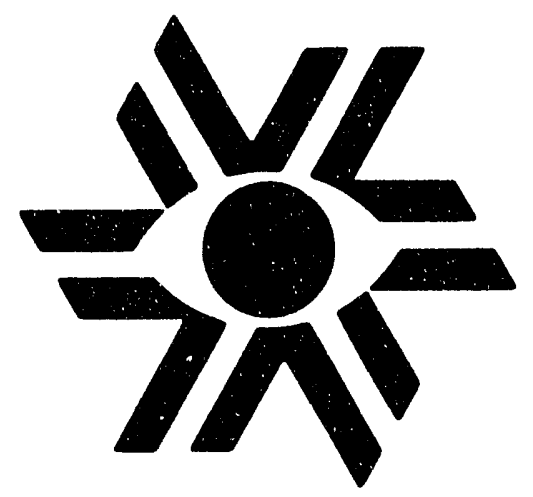

MAdison, Wisconsin 53706-1687 
This report has been reproduced directly from the best available copy.

Available to DOE and DOE contractors from the Office of Scientific and Technical Information, P.O. Box 62, Oak Ridge, TN 37831; prices available from (615) 576-8401, FTS 626-8401.

Available to the public from the National Technical Information Service, U.S. Department of Commerce, 5285 Port Royal Rd., Springfield, VA 22161.

This report was prepared as an account of work sponsored by an agency of the United States Government. Neither the United States Government nor any agency thereof, nor any of their employees, makes any warranty, express or implied, or assumes any legal liability or responsibility for the accuracy, completeness, or usefulness of any information, apparatus, product, or process disclosed, or represents that its use would not infringe privately owned rights. Reference herein to any specific commercial product, process, or service by tradename, trademark, manufacturer, or otherwise, does not necessarily constitute or imply its endorsement, recommendation, or favoring by the United States Government or any agency thereof. The views and opinions of authors expressed herein do not necessarily state or reflect those of the United States Government or any agency thereof. 


\title{
POLOIDAL VARIATION OF VISCOUS FORCES
} IN THE BANANA COLLISIONALITY REGIME

\author{
J. P. WANG AND J. D. CALLEN \\ Nuclear Engineering \& Engineering Physics Department \\ University of Wisconsin-Madison, \\ Madison, Wisconsin 53706-1687
}

UW-CPTC 92-6

December 1992

This is a preprint of a paper to be submitted for publication in The Physics of Fluids B

\section{MASTER}


December 1992

\title{
Poloidal Variation of Viscous Forces in the Banana Collisionality Regime
}

\author{
J.P. Wang and J.D. Callen \\ Nuclear Engineering \& Engineering Physics Department \\ University of Wisconsin-Madison \\ Madison, WI 53706-1687
}

The poloidal variation of the parallel viscous and heat viscous forces are determined for the first time using a rigorous Chapman-Enskog-like approach that has been developed recently. It is shown that the poloidal variation is approximately proportional to the poloidal distribution of the trapped particles, which are concentrated on the outer edge (large major radius side) of the tokamak.

PACS numbers: 51.10.+y, 52.25.Dg, 52.55.Fa, 52.30.-q 


\section{INTRODUCTION}

All "banana-plateau" neoclassical transport effects stem from the parallel forces due to the viscous stress along a magnetic field line. ${ }^{1}$ The neoclassical viscous forces in the low collisionality regime are usually derived somewhat indirectly, ${ }^{1,2}$ and are given as averages over a magnetic flux-surface. Recently, a rigorous Chapman-Enskog-like procedure ${ }^{3}$ has been developed for determining the kinetic equation for the kine'cic distortions of the distribution function from which the viscous stress; and parallel viscous forces can be calculated directly. ${ }^{4}$ In this paper we use this approach to determine, for the first time, the poloidal angle dependence of the parallel (to the magnetic field B) viscous force in the low, or banana collisionality regime.

In Ref. 3, the Fokker-Planck plasma kinetic equation was recast into an equation for the kinetic distortion $F$, which has a source driven by the stress tensors of mass flow and heat flux, $\nabla \mathrm{V}$ and $\nabla \mathrm{q}$, using the Ansatz:

$$
f=f_{M}\left[1-\frac{2}{v_{T}^{2}} \mathbf{w} \cdot\left(\frac{2}{5 n T} \mathbf{q}\right) L_{1}^{(3 / 2)}\right]+F .
$$

Here, $\mathbf{w} \equiv \mathbf{v}-\mathbf{V}$ represents the particle velocity measured in the reference frame moving with the fluid mass flow velocity. The recast equation is then gyro-phase averaged using a small gyro-radius approvimation, $\delta \sim \rho \nabla_{\perp} \ln n \sim \rho / \ell_{\perp} \ll 1$. The resultant Chapman-Enskog-like version of the drift kinetic equation can be expressed as follows [cf., Eq. (127) in Ref. 3]:

$$
\begin{aligned}
& \frac{\partial F}{\partial t}+{ }^{\prime}\left(w_{\|} \mathbf{b}+\mathrm{V}\right) \cdot \nabla F-C_{R}(F)= \\
& \left\{-\left(w_{\|}^{2}-\frac{w_{\perp}^{2}}{2}\right) G+w_{\|} \mathbf{b} \cdot \frac{1}{p}\left[L_{0}^{(3 / 2)} \nabla \cdot \Pi_{\|}\right.\right. \\
& \left.+\frac{2}{5} L_{1}^{(3 / 2)}\left(\nabla \cdot \Theta_{\|}-\frac{4}{3 T} \nabla\left(T^{2} \int d^{3} v L_{2}^{(1 / 2)}\langle F\rangle\right)\right)\right] \\
& \left.\quad-\frac{8}{15 p} \nabla \cdot \mathrm{q} L_{2}^{(1 / 2)}\right\} f_{M} \\
& G=\frac{m}{T}\left\{\left[\left(\mathrm{~V}+\frac{2}{5 p} \mathrm{q}\right) \cdot \nabla \ln B-\frac{1}{B} \mathrm{~b} \cdot \nabla \times(\mathrm{B} \times \mathrm{V})\right.\right.
\end{aligned}
$$




$$
\begin{aligned}
& \left.-\frac{2}{5 p} \frac{1}{B} \mathbf{b} \cdot \nabla \times(\mathbf{B} \times \mathbf{q})+\frac{2}{3}\left(\nabla \cdot \mathrm{V}+\frac{2}{5 p} \nabla \cdot \mathbf{q}\right)\right] L_{0}^{(5 / 2)} \\
& \left.-\frac{2}{5 p}\left[\mathbf{q} \cdot \nabla \ln B+\frac{2}{3} \nabla \cdot \mathbf{q}-\frac{1}{B} \mathbf{b} \cdot \nabla \times(\mathbf{B} \times \mathbf{q})\right] L_{1}^{(5 / 2)}\right\} .
\end{aligned}
$$

Here, all the gyro-averaging notation has been neglected for simplicity, $b \equiv$ $\mathbf{B} / B$ is a unit vector along the magnetic field, $w_{\|}$represents the parallel (to the magnetic field $\mathbf{B}$ ) component of the particle random velocity. The collisional term above is given by:

$$
C_{R}(F)=\frac{1}{2} \nu_{\perp}(w) £ F+w_{\|}\left(\nu_{0} L_{0}^{(3 / 2)}+\nu_{1} L_{1}^{(3 / 2)}\right) f_{M}
$$

where $£$ is the Lorentz scattering operator and the "collisional restoring" (field-particle response) coefficients are defined by

$$
\nu_{0}=-\frac{m}{p}\left[\frac{2 q_{\|}}{5 p} \int \frac{\nu_{\perp}}{3} \frac{w^{2}}{v_{T}^{2}} L_{1}^{(3 / 2)} f_{M} d^{3} v+\int \frac{\nu_{\perp}}{2} w_{\|} L_{0}^{(3 / 2)} £ F d^{3} v\right]
$$

and

$$
\begin{aligned}
\nu_{1}= & \frac{2 q_{\|}}{5 p} \frac{\nu_{\perp}(w)}{v_{T}^{2}}-\frac{2 m}{5 p}\left[\frac{2 q_{\|}}{5 p} \int \frac{\nu_{\perp}}{3} \frac{w^{2}}{v_{T}^{2}}\left(L_{1}^{(3 / 2)}\right)^{2} f_{M} d^{3} v\right. \\
& \left.+\int \frac{\nu_{\perp}}{2} w_{\|} L_{1}^{(3 / 2)} £ F d^{3} v\right] .
\end{aligned}
$$

Everything else is the same as that defined in Ref 3. The above kinetic equation should be solved subject to the constraints:

$$
\int d^{3} v\left(L_{0}^{(1 / 2)}, L_{1}^{(1 / 2)}\right) F=0
$$

and

$$
\int d^{3} v\left(w_{\|} L_{0}^{(3 / 2)}, w_{\|} L_{1}^{(3 / 2)}\right) F=0
$$

since the kinetic distortion must not contribute to the fluid moments, density, temperature, mass flow and heat flux according to the Ansatz given by Eq. (1).

The rest of this paper is organized as follows. In Sec. II, we introduce various approximations based on the fluid moment equations, such as the 
traditional mass flow and heat flux incompressibilities adopted in neoclassical theory, and thereby simplify the drift kinetic equation given by Eqs. (2) and (3). In Sec. III, the lowest order drift kinetic solution is obtained assuming a subsidiary large bounce and small collision frequency ordering valid in the fusion-relevant low-collisionality ("banana") regime. In Sec. IV, the parallel viscous stress forces $\mathbf{B} \cdot \nabla \cdot \boldsymbol{I I}_{\|}$and $\mathbf{B} \cdot \nabla \cdot \Theta_{\|}$are derived by taking the proper moments of the first order, $\mathcal{O}\left(\delta \nu_{*}\right)$, Chapman-Enskog-like version of the drift kinetic equation. The poloidal angle dependence is also determined through this direct procedure. Sec. V discusses the results achieved and their implications.

\section{MOMENT APPROXIMATIONS}

\section{A. Mass flow incompressibility}

Taking the cross product of the momentum balance equation [given by Eq. (15) in Ref. 3] with the magnetic field B, we can obtain the perpendicular fluid velocity for a given plasma species

$$
\mathrm{V}_{\perp}=\frac{c \mathbf{E} \times \mathbf{B}}{B^{2}}-\frac{c \nabla p \times \mathbf{B}}{n q B^{2}}-\frac{c \nabla \cdot \Pi \times \mathbf{B}}{n q B^{2}}+\frac{c \mathbf{F}_{0} \times \mathbf{B}}{n q B^{2}}+\frac{m c}{q B^{2}} \mathrm{~B} \times \frac{D \mathrm{~V}}{D t}
$$

Here, the first and second term represent the first order " $\mathrm{E} \times \mathrm{B}$ " and diamagnetic flows respectively, the third and fourth terms represent the second order (in $\delta$ ) neoclassical and classical transport, respectively, and the last term represents either a third or second order polarization drift depending on whether the slower diffusion or a faster time scale is considered. Although the classical, neoclassical and polarization drift flows are second order in $\delta$, we need only take account of the first order effects in determining the kinetic distortion $F$ whose moments yield the desired stress tensor $\Pi_{\|}$and hea.t stress tensor $\Theta_{\|}$. Thus to the lowest significant order $(\delta)$, we obtain

$$
\mathbf{V}_{\perp}=\frac{c \mathbf{E} \times \mathbf{B}}{B^{2}}-\frac{c \nabla p \times \mathbf{B}}{n q B^{2}}
$$


Deploying the diffusion time scale assumption, $\partial / \partial t \sim \mathcal{O}\left(\delta^{2}\right)$, we obtain

$$
\nabla \cdot \mathrm{V} \simeq-\frac{1}{n}\left(V_{\|} \mathrm{b}+\mathrm{V}_{\perp}\right) \cdot \nabla \psi \frac{\partial n_{0}}{\partial \psi}
$$

from the density conservation equation (to the lowest order)

$$
\frac{\partial n}{\partial t}+\nabla \cdot(n \mathrm{~V})=0
$$

Using Eq. (10) for the perpendicular flow and assuming that the electric field is mainly electrostatic, Eq. (11) yields the important incompressibility result

$$
\nabla \cdot \mathrm{V} \simeq 0
$$

utilized in neoclassical transport studies. ${ }^{1}$ We restrict ourselves to an axisymmetric toroidal magnetic field expressed as

$$
\mathbf{B}=I \nabla \zeta+\nabla \zeta \times \nabla \psi
$$

where $|\nabla \psi|=R B_{p}$ and $I(\psi)=R B_{t}$ in which $B_{p}$ and $B_{t}$ represent the poloidal and toroidal magnetic field, respectively. Using Eq. (10) and the electrostatic assumption for $V_{\perp}$, we can cast the divergence of the mass flow into the form

$$
\nabla \cdot \mathbf{V}=\mathbf{B} \cdot \nabla\left[\frac{V_{\|}}{B}+\frac{c I}{B^{2}}\left(\frac{\partial \phi}{\partial \psi}+\frac{1}{n q} \frac{\partial p}{\partial \psi}\right)\right]
$$

Here, we have used the fact that

$$
(\nabla \times \mathrm{B}) \cdot \nabla \psi=0
$$

since no current should cross a magnetic flux surface in a ideal magnetohydrodynamic equilibrium. It is easy to recognize that the expression in the square bracket in Eq. (15) is just the "poloidal flow" defined by

$$
\begin{aligned}
u_{\theta} & \equiv \frac{\mathrm{V} \cdot \nabla \theta}{\mathrm{B} \cdot \nabla \theta} \\
& =\frac{V_{\|}}{B}+\frac{c I}{B^{2}}\left(\frac{\partial \phi}{\partial \psi}+\frac{1}{n q} \frac{\partial p}{\partial \psi}\right) .
\end{aligned}
$$

Considering the incompressibility given by Eq. (13), the above results [i.e. Eqs. (15) and (17)] imply that the "poloidal flow" $u_{0}$ is solely a function of the flux surface variable $\psi$ :

$$
u_{\theta}=u_{\theta}(\psi)
$$




\section{B. Heat flux incompressibility}

Similarly, cross multiplying the heat flow balance equation [given by Eq. (16) in Ref. 3], we can obtain the first order [in $\delta$ ] perpendicular heat flux

$$
\mathrm{q}_{\perp} \simeq \frac{5}{2} \frac{c p \mathrm{~B} \times \nabla T}{q B^{2}}
$$

On the diffusive time scale we can derive the heat flux incompressibility

$$
\nabla \cdot \mathbf{q} \simeq-\frac{3 p}{2}\left(V_{\|} \mathbf{b}+\mathrm{V}_{\perp}\right) \cdot \nabla \psi \frac{\partial \ln T_{0}}{\partial \psi} \simeq 0
$$

valid to first order in $\delta$, utilizing the energy conservation equation given by Eq. (4) in Ref. 3 and Eqs. (19) and (13). Substituting in Eq. (19) for the lowest order perpendicular heat flux, we find

$$
\nabla \cdot \mathbf{q}=\mathbf{B} \cdot \nabla\left[\frac{q_{\|}}{B}+\frac{5 c I p}{2 q B^{2}} \frac{\partial T}{\partial \psi}\right]
$$

where the terms in the square bracket above can be easily recognized as the "poloidal heat flux" defined by

$$
\begin{aligned}
q_{\theta} & \equiv \frac{\mathbf{q} \cdot \nabla \theta}{\mathbf{B} \cdot \nabla \theta} \\
& =\frac{q_{\|}}{B}+\frac{5 c I p}{2 q B^{2}} \frac{\partial T}{\partial \psi}
\end{aligned}
$$

Then, it is obvious that the poloidal heat flux is constant within a magnetic flux surface, i.e.,

$$
q_{\theta}=q_{\theta}(\psi)
$$

considering the heat flux incompressibility given by Eq. (20).

\section{Reduction of kinetic equation}

Using the diffusion time scale and incompressibility approximations given by Eqs. (13) and (20), the Chapman-Enskog-like version of the drift kinetic 
equation given in Eq. (2) can be simplified to

$$
\begin{aligned}
& w_{\|} \mathbf{b} \cdot \nabla F-C_{R}(F)= \\
& \quad\left[-\left(w_{\|}^{2}-\frac{w_{\perp}^{2}}{2}\right) G+\frac{w_{\|}}{p} \mathbf{b} \cdot\left(L_{0}^{(3 / 2)} \nabla \cdot \Pi_{\|}+\frac{2}{5} L_{1}^{(3 / 2)} \nabla \cdot \Theta_{\|}\right)\right] f_{M} .
\end{aligned}
$$

Here, the Legendre polynomial $P_{2}\left(w_{\|} / w\right)$-type coefficient has been simplified to

$$
G \simeq \frac{m}{T}\left\{\left[\left(\mathrm{~V}+\frac{2}{5 p} \mathrm{q}\right) \cdot \nabla \ln B\right] L_{0}^{(5 / 2)}-\frac{2}{5 p}(\mathrm{q} \cdot \nabla \ln B) L_{1}^{(5 / 2)}\right\},
$$

since we can easily verify that

$$
\begin{aligned}
& \nabla \times(\mathrm{B} \times \mathrm{V}) \simeq 0 \\
& \nabla \times(\mathrm{B} \times \mathrm{q}) \simeq 0
\end{aligned}
$$

using Eqs. (10) ard (19). The b. $\nabla\left(T^{2} \int d^{3} v L_{2}^{(1 / 2)} F\right)$ term in Eq. (2) has been neglected because it is a higher order energy weighted moment and does not lead to net radial transport. Because the lowest order mass flow and heat flux are constant on the flux surface, we find

$$
\begin{aligned}
\mathbf{V} \cdot \nabla \ln B & =\frac{\mathbf{V} \cdot \nabla \theta}{\mathbf{B} \cdot \nabla \theta} \mathbf{B} \cdot \nabla \theta \frac{\partial \ln B}{\partial \theta} \\
& =u_{\theta} \mathbf{B} \cdot \nabla \ln B \\
\mathbf{q} \cdot \nabla \ln B & =\frac{\mathbf{q} \cdot \nabla \theta}{\mathbf{B} \cdot \nabla \theta} \mathbf{B} \cdot \nabla \theta \frac{\partial \ln B}{\partial \theta} \\
& =q_{\theta} \mathbf{B} \cdot \nabla \ln B .
\end{aligned}
$$

Therefore, the $P_{2}$-type coefficient $G$ can be expressed as

$$
G=\frac{m}{T}\left[\left(u_{\theta}+\frac{2}{5 p} q_{\theta}\right) L_{0}^{(5 / 2)}-\frac{2}{5 n} q_{\theta} L_{1}^{(5 / 2)}\right] \mathrm{B} \cdot \nabla \ln B .
$$

Keeping in mind that the independent variables ior the drift kinetic equation are $t, \mathbf{x}, \mu$ and $w$, we can cast the $P_{2}$-type driven source into a free streaming form:

$$
\left(w_{\|}^{2}-\frac{w_{\perp}^{2}}{2}\right) G=w_{\|} \mathbf{b} \cdot \nabla\left[w_{\|} B \frac{m}{T}\left(u_{\theta} L_{0}^{(3 / 2)}-\frac{2}{5 p} q_{0} L_{1}^{(3 / 2)}\right) f_{M}\right]
$$


utilizing the relation

$$
w_{\|} \mathbf{b} \cdot \nabla\left(w_{\|} B\right)=\left(w_{\|}^{2}-\frac{w_{\perp}^{2}}{2}\right) \mathbf{B} \cdot \nabla \ln B .
$$

As we will see below, this conversion is essential in deriving the low collisionality regime viscous forces. Here, we have also used the fact that

$$
\mathbf{b} \cdot \nabla f(\psi)=0
$$

for an arbitrary function of the magnetic flux surface variable $\psi$, and converted the Laguerre polynomials according to $L_{0}^{(5 / 2)}=L_{0}^{(3 / 2)}$ and $L_{1}^{(5 / 2)}=$ $L_{0}^{(3 / 2)}+L_{1}^{(3 / 2)}$. Thus, the drift kinetic equation can be further reduced to the following form

$$
\begin{aligned}
w_{\|} \mathbf{b} \cdot \nabla & {\left[F+w_{\|} B \frac{m}{T}\left(u_{\theta} L_{0}^{(3 / 2)}-\frac{2}{5 p} q_{0} L_{1}^{(3 / 2)}\right) f_{M}\right]-\frac{\nu_{\perp}}{2} £ F=} \\
w_{\|} & {\left[\left(\nu_{0}+\frac{1}{p} \mathbf{b} \cdot \nabla \cdot \Pi_{\|}\right) L_{0}^{(3 / 2)}+\left(\nu_{1}+\frac{2}{5 p} \mathbf{b} \cdot \nabla \cdot \Theta_{\|}\right) L_{\perp}^{(3 / 2)}\right] f_{M} }
\end{aligned}
$$

by substituting Eqs. (4) and (31) into Eq. (24), where the collisional coefficients $\nu_{0}$ and $\nu_{1}$ have been given in Eqs. (5) and (6), respectively.

\section{BANANA REGIME DRINT KINETICS}

\section{A. Rapid bounce motion approximation}

The bouncing/circulating motion operator in Eq. (34) can be written as

$$
w_{\|} \mathbf{b} \cdot \nabla=w_{\|} \mathbf{b} \cdot \nabla \theta \frac{\partial}{\partial \theta} \sim \frac{v_{\|}}{v} \frac{v}{R_{0} q_{s}}
$$

while the collisional pitch-angle scattering term can be ordered as

$$
\frac{\nu_{\perp}}{2} £ \sim \frac{\nu}{\left(v_{\|} / v\right)^{2}}
$$


In the low collisionality regime (often referred to as the banana regime), the bounce frequency is much larger than the collision frequency ${ }^{1,5}$ - trapped and untrapped particles can complete their banana and circulating orbits before collisions occur. Since the typical pitch-angle for trapped particles is $v_{\|} / v \sim \sqrt{\epsilon}$, we adopt the large bounce, low collision frequency assumption

$$
\nu_{*} \equiv \frac{\nu}{\epsilon^{3 / 2} \omega_{b}} \ll 1
$$

valid in the banana collisionality regime. Here, we have defined the untrapped particle bounce frequency

$$
\therefore=\frac{v_{T}}{R_{0} g_{s}}
$$

in which $q_{s}$ and $R_{0}$ represent the safety factor and major radius, respectively.

Then, the kinetic distortion $F$ can be expanded in the subsidiary small parameter $\nu_{*}: F=F_{0}+\nu_{*} F_{1}+\cdots \cdot$. To the lowest order in the subsidiary small parameter $\left[\mathcal{O}\left(\delta \nu_{*}^{0}\right)\right.$ to be specific], we find that the drift kinetic equation becomes

$$
w_{\|} \mathbf{b} \cdot \nabla\left\{F_{0}+\left[w_{\|} B \frac{m}{T}\left(u_{\theta} L_{0}^{(3 / 2)}-\frac{2 q_{\theta}}{5 p} L_{1}^{(3 / 2)}\right)\right] f_{M}\right\}=0
$$

since the viscous stress and heat viscous stress are higher order collisional effects. In the next order, $\mathcal{O}\left(\delta \nu_{*}\right)$, approximation, the drift kinetic equation can be written as

$$
\begin{aligned}
& w_{\|} \mathbf{b} \cdot \nabla F_{1}-\frac{\nu_{\perp}}{2} £ F_{0}= \\
& \quad w_{\|}\left[\left(\nu_{0}+\frac{1}{p} \mathbf{b} \cdot \nabla \cdot \Pi_{\|}\right) L_{0}^{(3 / 2)}+\left(\nu_{1}+\frac{2}{5 p} \mathbf{b} \cdot \nabla \cdot \Theta_{\|}\right) L_{1}^{(3 / 2)}\right] f_{M} .
\end{aligned}
$$

The collisional coefficients $\nu_{0}$ and $\nu_{1}$ are determined to lowest order from the solution for $F_{0}$ as follows

$$
\nu_{0}=-\frac{m}{p}\left[\frac{2 q_{\|}}{5 p} \int \frac{\nu_{\perp}}{3} \frac{w^{2}}{v_{T}^{2}} L_{0}^{(3 / 2)} L_{1}^{(3 / 2)} f_{M} d^{3} v+\int \frac{\nu_{\perp}}{2} w_{\|} L_{0}^{(3 / 2)} £ F_{0} d^{3} v\right]
$$

and

$\nu_{1}=\frac{2 q_{\|}}{5 p} \frac{\nu_{\perp}(w)}{v_{T}^{2}}-\frac{2 m}{5 p}\left[\frac{2 q_{\|}}{5 p} \int \frac{\nu_{\perp}}{3} \frac{w^{2}}{v_{T}^{2}}\left(L_{1}^{(3 / 2)}\right)^{2} f_{M} d^{3} v+\int \frac{\nu_{\perp}}{2} w_{\|} L_{1}^{(3 / 2)} £ F_{0} d^{3} v\right]$, 
according to Eqs. (5) and (6). Here, the last term in the above two equations can be shown vanish later [refer to Eq. (58) below]. The Lorentz collision operator can be expressed as

$$
£ F_{0}=w_{\|} \frac{\partial}{\partial \mu}\left(\frac{\mu w_{\|}}{B} \frac{\partial F_{0}}{\partial \mu}\right)
$$

using the relative velocity $w_{\|}$, and random magnetic moment $\mu$ as the independent velocity space variables.

\section{B. Lowest order drift kinetic solution}

It is obvious that the solution of Eq. (37) is of the form

$$
F_{0}=-w_{\|} B \frac{m}{T}\left(u_{0} L_{0}^{(3 / 2)}-\frac{2}{5 p} q_{\theta} L_{1}^{(3 / 2)}\right) f_{M}+g(w, \mu, \psi, \sigma) .
$$

Here, the solubility constraint Eq. (7) has been applied to eliminate the possible presence of a $P_{0}$-type term from the solution for $F_{0}$, and $\sigma$ has been defined as the sign of the parallel velocity. The integral constant $g$ can be determined by taking the bounce average of the first order drift kinetic equation using the annihilator $1,6,7$

$$
\oint \frac{d l}{v_{\|}} \equiv \begin{cases}\int_{0}^{2 \pi} \frac{d \theta}{v_{\|} \mathrm{b} \cdot \nabla \theta}, & \text { circulating particles } \\ \sum_{\sigma} \int_{-\theta_{-}}^{+\theta_{c}} \frac{d \theta}{\left|v_{\|}\right| \mathrm{b} \cdot \nabla \theta}, & \text { trapped particles }\end{cases}
$$

and the solubility constraint given in Eq. (8). Here, $\theta_{c}$ represents the turning point of the trapped particles. Applying the annihilator for trapped particles to Eq. (38) and noticing that

$$
\oint \frac{1 l}{v_{\|}} \simeq \oint \frac{d l}{w_{\|}}
$$

to the lowest order, we obtain

$$
\frac{\partial g_{t}}{\partial \mu}=\frac{C}{\mu \int_{-o_{c}}^{+\theta_{c}} \frac{d 6}{\mathbf{B} \cdot \nabla \theta}\left|w_{\|}\right|}
$$


To remove the singularity at $\mu=0$, we choose $C=0 .{ }^{6}$ Also, considering the density conservation at the turning point $g_{t}(\sigma)=g_{t}(-\sigma)$, we obtain ${ }^{6}$

$$
g_{t}=0
$$

Similarly, applying the annihilator for untrapped particles to Eq. (38), we obtain

$$
g_{u t} \sim \int_{\mu}^{\mu_{\mathrm{c}}} \frac{d \mu}{\left\langle w_{\|}\right\rangle}
$$

where

$$
\mu_{c}=\frac{w_{\perp}^{2}}{2 B_{\max }}
$$

and the angle bracket represents the flux surface average defined by

$$
\langle f\rangle \equiv\left(\int_{0}^{2 \pi} \frac{d \theta}{\mathrm{B} \cdot \nabla \theta}\right)^{-1} \int_{0}^{2 \pi} \frac{d \theta}{\mathrm{B} \cdot \nabla \theta} f .
$$

It is convenient to introduce a unit step function

$$
H\left(\lambda_{c}-\lambda\right)=\left\{\begin{array}{lll}
1, & \lambda<\lambda_{c}, & \text { circulating } \\
0, & \lambda \geq \lambda_{c}, & \text { trapped }
\end{array}\right.
$$

Then, the function $g$ can be expressed as

$$
g=g_{u t} H\left(\lambda_{c}-\lambda\right)
$$

where $\lambda=2 \mu / 2 w^{2}, \lambda_{c} \equiv 1 / B_{\max }$. Note also that in terms of these variables $w_{i}=w \sigma \sqrt{1-\lambda B}$. Recalling that the distribution function $F$ does not contribute to the mass flow and heat flux [see the solubility constraint given in Eq. (8)], we find that

$$
g=\frac{\left\langle B^{2}\right\rangle}{f_{c}} \frac{m}{T}\left(u_{\theta} L_{0}^{(3 / 2)}-\frac{2 g_{\theta}}{5 p} L_{1}^{(3 / 2)}\right) f_{M} \frac{\sigma w}{2} \int_{\lambda}^{\lambda_{c}} \frac{d \lambda^{\prime}}{\left\langle\sqrt{1-\lambda^{\prime} B}\right\rangle} H\left(\lambda_{c}-\lambda\right)
$$

Here, the "flow fraction" of circulating particles is defined as ${ }^{1}$

$$
f_{c} \equiv \frac{3}{4}\left\langle B^{2}\right\rangle \int_{0}^{\lambda_{c}} \frac{\lambda^{\prime} d \lambda^{\prime}}{\left\langle\sqrt{1-\lambda^{\prime} B}\right\rangle} .
$$


Since the lowest order solution $F_{0}$ is an odd function of $\sigma \equiv \operatorname{sign}\left(w_{\|}\right)$, to determine the viscous stresses it is necessary to go to the next order $\mathcal{O}\left(\nu_{*}\right)$ in the drift kinetic equation. First, let us define a velocity-like variable

$$
u_{\|} \equiv \frac{\sigma w}{2} \int_{\lambda}^{\lambda_{c}} \frac{d \lambda^{\prime}\left(B^{2}\right\rangle^{1 / 2}}{\left\langle\sqrt{1-\lambda^{\prime} B}\right\rangle} H\left(\lambda_{c}-\lambda\right),
$$

which may be interpreted as the circulating particle flow along a magnetic field line in an inhomogeneous magnetic field. This definition differs with Hirshman Sigmar's definition ${ }^{1}$ by a unit step function factor which has been introduced to keep it well defined. Thus, the lowest order kinetic distortion can be conveniently expressed as

$$
F_{0}=-\left(w_{\|} B-\frac{\left\langle B_{i}^{2}{ }^{1 / 2}\right.}{f_{c}} u_{\|}\right) \frac{m}{T}\left(u_{0} L_{0}^{(3 / 2)}-\frac{2}{5 p} q_{0} L_{1}^{(3 / 2)}\right) f_{M} .
$$

For convenience in later calculations, let us consider an integral property first. Suppose that $f(w)$ is an arbitrary isotropic function in velocity space. Then, using the fact that ${ }^{6}$

$$
\int d^{3} v=\sum_{\sigma} \pi \int_{0}^{\infty} w^{3} d w \int_{0}^{1 / B} \frac{d \lambda B}{\left|w_{\|}\right|}
$$

upon choosing $w$ and $\lambda$ as independent variables, we find:

$$
\begin{aligned}
& \int d^{3} v w_{\|} u_{\|} f(w) \\
& =\sum_{\sigma} \pi \int_{0}^{\infty} w^{3} f(w) d w \int_{0}^{1 / B} \frac{d \lambda B}{\left|w_{\|}\right|} w_{\|} u_{\|} \\
& =B \sum_{\sigma} \pi \int_{0}^{\infty} \frac{w^{4}}{2} f(w) d w \int_{0}^{1 / B} d \lambda \int_{\lambda}^{\lambda_{c}} \frac{\left\langle B^{2}\right\rangle^{1 / 2} d \lambda^{\prime}}{\left\langle\sqrt{1-\lambda^{\prime} B}\right\rangle} H\left(\lambda_{c}-\lambda\right) \\
& =B \int d^{3} v^{\prime} \frac{w^{2}}{4} f(w) \int_{0}^{\lambda_{c}} \frac{\lambda d \lambda\left\langle B^{2}\right\rangle^{1 / 2}}{\langle\sqrt{1-\lambda B}\rangle} \\
& =\frac{B f_{c}}{\left\langle B^{2}\right\rangle^{1 / 2}} \int d^{3} v \frac{w^{2}}{3} f(w) .
\end{aligned}
$$

Operating on the $F_{0}$ in Eq. (54) with the Lorentz collision operator given by Eq. (41), we obtain

$$
£ F_{0}=\left(\frac{\left\langle B^{2}\right\rangle^{1 / 2}}{f_{c}} £ u_{\|}+w_{\|} B\right) \frac{m}{T}\left(u_{0} L_{0}^{(3 / 2)}-\frac{2}{5 p} q_{\theta} L_{1}^{(3 / 2)}\right) f_{M}
$$


Then, utilizing Eqs. (41), (54) and (55), we find

$$
\begin{aligned}
& \int \frac{\nu_{\perp}}{2} w_{\|} L_{i}^{3 / 2} £ F_{0} d^{3} v \\
& =\frac{\left\langle B^{2}\right\rangle^{1 / 2}}{f_{c}} \int d^{3} v \frac{\nu_{\perp}}{v_{T}^{2}}\left(u_{0} L_{0}^{(3 / 2)}-\frac{2}{5 p} q_{\theta} L_{1}^{(3 / 2)}\right) L_{i}^{(3 / 2)} f_{M} w_{\|} \mathcal{L} u_{\|} \\
& \quad+B \int d^{3} v \frac{\nu_{\perp}}{v_{T}^{2}} \frac{v^{2}}{3}\left(u_{0} L_{0}^{(3 / 2)}-\frac{2}{5 p} q_{0} L_{1}^{(3 / 2)}\right) L_{i}^{(3 / 2)} f_{M} \\
& =0 .
\end{aligned}
$$

Here, we have integrated by parts over the $\lambda$ variable. Therefore, the viscous coefficients $\nu_{0}$ and $\nu_{1}$ can be simplified to the following form in banana regime:

$$
\begin{gathered}
\nu_{0}=-\frac{m}{p} \frac{2 q_{\|}}{5 p} \int \frac{\nu_{\perp}}{3} \frac{w^{2}}{v_{\tau}^{2}} L_{0}^{(3 / 2)} L_{1}^{(3 / 2)} f_{M} d^{3} v \\
\nu_{1}=\frac{2 q_{\|}}{5 p} \frac{\nu_{\perp}(w)}{v_{T}^{2}}-\frac{2 m}{5 p} \frac{2 q_{\|}}{5 p} \int \frac{\nu_{\perp}}{3} \frac{w^{2}}{v_{T}^{2}}\left(L_{1}^{(3 / 2)}\right)^{2} f_{M} d^{3} v .
\end{gathered}
$$

These integrals can be readily performed, as indicated in Appendix A.

\section{PARALLEL VISCOUS STRESS FORCES}

Having obtained the lowest order drift kinetic solution, we are now ready to derive the parallel viscous and heat viscous forces, $\left\langle\mathbf{B} \cdot \nabla \cdot \Pi_{\|}\right\rangle$and $\left\langle\mathbf{B} \cdot \nabla \cdot \Theta_{\|}\right\rangle$, directly by taking proper integral moments of the first order drift kinetic equation, Eq. (38). In Ref. 4, the flux-surface-averaged viscous forces, $\left\langle\mathbf{B} \cdot \nabla \cdot \Pi_{\|}\right\rangle$ and $\left\langle\mathbf{B} \cdot \nabla \cdot \Theta_{\|}\right\rangle$, were derived from the bounce averaged Chapman-Enskog-like version of the drift kinetic equation by taking the so called "proper" moments of the form $\int d^{3} v w_{\|} u_{\|} L_{i}^{(3 / 2)}$. This procedure averages over the poloidal angle variation. Utilizing the Chapman-Enskog-like procedure developed in Ref. 3 and the preceding analysis, we will now determine the poloidal angle dependence of the viscous forces in the banana collisionality regime.

Since the mass flow and heat flux moment equations have already been used in recasting the kinetic equation, taking the $\left(w_{\|} L_{0}^{(3 / 2)}, w_{\|} L_{1}^{(3 / 2)}\right)$ moments of the Chapman-Enskog-like version of the drift kinetic equation yields 
only null identities. Looking at the structure of Eq. (33), which determines the viscous forces, it is found that taking

$$
\int a^{3} v \frac{\left\langle B^{2}\right\rangle^{1 / 2}}{f_{c}} u_{\|}\left[\begin{array}{c}
L_{0}^{(3 / 2)} \\
L_{1}^{(3 / 2)}
\end{array}\right]
$$

moments of the first order Chapman-Enskog-like version of the drift kinetic equation given in Eq. (38) is most convenient for deriving the poloidal variation of the viscous forces without having to explicitly solve for the distribution function $F_{1}$. These moments of the first term on the left side of Eq. (38) yield

$$
\begin{aligned}
& \int \dot{u}^{3} v \frac{\left\langle B^{2}\right\rangle^{1 / 2}}{f_{c}} u_{\|}\left[\begin{array}{c}
L_{0}^{(3 / 2)} \\
L_{1}^{(3 / 2)}
\end{array}\right] w_{\|} \mathbf{b} \cdot \nabla F_{1} \\
& =\sum_{\sigma} \pi w^{3} d w \int_{0}^{\lambda_{c}} \frac{d \lambda B}{\left|w_{\|}\right|} w_{\|} \mathbf{b} \cdot \nabla \frac{\left\langle B^{2}\right\rangle^{1 / 2}}{f_{c}} u_{\|} F_{1}\left[\begin{array}{c}
L_{0}^{(3 / 2)} \\
L_{1}^{(3 / 2)}
\end{array}\right] \\
& =\frac{\left\langle B^{2}\right\rangle^{1 / 2}}{f_{c}} \mathbf{B} \cdot \nabla\left(\frac{1}{B} \int d^{3} v w_{\|} u_{\|} F_{1}\left[\begin{array}{c}
L_{0}^{(3 / 2)} \\
L_{1}^{(3 / 2)}
\end{array}\right]\right),
\end{aligned}
$$

where the integration over $\lambda$ goes from zero to the trapping boundary $\lambda_{c}$ because of the unit step function in the definition of $u_{\|}$. These two moments are of the differential form $\sim \frac{\partial}{\partial \theta}(\cdots)$ and thus vanish upon averaging over the poloidal flux surface.

The moments of the second term on the left side of Eq. (38) result in

$$
\begin{aligned}
& \int d^{3} v \frac{\left\langle B^{2}\right\rangle^{1 / 2}}{f_{c}} u_{\|}\left[\begin{array}{c}
L_{0}^{(3 / 2)} \\
L_{1}^{(3 / 2)}
\end{array}\right] \frac{\nu_{\perp}}{2} £ F_{0} \\
& =\sum_{\sigma} 2 \pi \int_{0}^{\infty} w d w \frac{\nu_{\perp}}{2} \frac{\left\langle B^{2}\right\rangle^{1 / 2}}{f_{c}}\left[\begin{array}{c}
L_{0}^{(3 / 2)} \\
L_{1}^{(3 / 2)}
\end{array}\right] \int_{0}^{\lambda_{c}} d \lambda u_{\|} \frac{\partial}{\partial \lambda}\left(\lambda\left|u_{\|}\right| \frac{\partial F_{0}}{\partial \lambda}\right) .
\end{aligned}
$$

Substituting in Eqs. (53) and (54) for $u_{\|}$and $F_{0}$ respectively and integrating by parts, we obtain

$$
\int d^{3} v \frac{\left\langle B^{2}\right\rangle^{1 / 2}}{f_{c}} u_{\|}\left[\begin{array}{c}
L_{0}^{(3 / 2)} \\
L_{1}^{(3 / 2)}
\end{array}\right] \frac{\nu_{\perp}}{2} £ F_{0}
$$




$$
\begin{aligned}
= & \frac{\left\langle B^{2}\right\rangle}{f_{c}} \sum_{\sigma} 2 \pi \int_{0}^{\infty} w^{2} / w \frac{\nu_{\perp}}{2} \frac{w}{2}\left[\begin{array}{c}
L_{0}^{(3 / 2)} \\
L_{1}^{(3 / 2)}
\end{array}\right] \int_{0}^{\lambda_{c}} d \lambda \sigma \sqrt{1-\lambda B} \frac{\partial F_{0}}{\partial \lambda} \frac{\lambda d \lambda}{\langle\sqrt{1-\lambda B}\rangle} \\
= & \frac{\left\langle B^{2}\right\rangle}{f_{c}} \int d^{3} v \frac{\nu_{\perp}}{v_{T}^{2}} \frac{w^{2}}{3}\left(u_{\theta} L_{0}^{(3 / 2)}-\frac{2 q_{\theta}}{5 p} L_{1}^{(3 / 2)}\right)\left[\begin{array}{c}
L_{0}^{(3 / 2)} \\
L_{1}^{(3 / 2)}
\end{array}\right] f_{M} \\
& {\left[\frac{B^{2} f_{c}}{\left\langle B^{2}\right\rangle}-\frac{3\left\langle B^{2}\right\rangle}{4 f_{c}} \int_{0}^{\lambda_{c}} \frac{\sqrt{1-\lambda B}}{\langle\sqrt{1-\lambda B}\rangle} \frac{\lambda d \lambda}{\langle\sqrt{1-\lambda B}\rangle}\right] . }
\end{aligned}
$$

The energy integrals above can be more compactly expressed in terms of the viscosity coefficients (refer to Appendix A)

$$
\mu_{i j} \equiv \frac{1}{n} \frac{f_{t}}{f_{c}} \int d^{3} v \frac{\nu_{\perp}}{v_{T}^{2}} \frac{w^{2}}{3} L_{i}^{(3 / 2)} L_{j}^{(3 / 2)} f_{M}
$$

where $f_{t} \equiv 1-f_{c}$ represents the flow-weighted trapped particle fraction, which can be analytically evaluated in the large aspect ratio limit $(\epsilon \ll 1)^{6}$ :

$$
f_{t} \simeq 1.46 \sqrt{\epsilon}+\mathcal{O}\left(\epsilon^{3 / 2}\right)
$$

The poloidal angle dependence in Eq. (64) can be conveniently expressed by the following spatial shape factor

$$
\hat{f}_{i}(\theta) \equiv \frac{1}{f_{t}}\left[\frac{3\left\langle B^{2}\right\rangle}{4 f_{c}} \int_{0}^{\lambda_{c}} \frac{\sqrt{1-\lambda B}}{\langle\sqrt{1-\lambda B}\rangle} \frac{\lambda d \lambda}{\langle\sqrt{1-\lambda B}\rangle}-\frac{B^{2} f_{c}}{\left\langle B^{2}\right\rangle}\right],
$$

which, upon recalling the definition of $f_{c}$ given in Eq. (52) obviously yields unity upon a flux surface averaging operation:

$$
\left\langle\hat{f}_{t}(\theta)\right\rangle=1
$$

Therefore, Eq. (64) can be written as

$$
\begin{aligned}
& \int d^{3} v \frac{\left\langle B^{2}\right\rangle^{1 / 2}}{f_{c}} u_{\|}\left[\begin{array}{c}
L_{0}^{(3 / 2)} \\
L_{1}^{(3 / 2)}
\end{array}\right] \frac{\nu_{\perp}}{2} £ F_{0} \\
& =-n\left\langle B^{2}\right\rangle \hat{f}_{t}(\theta)\left[\begin{array}{ll}
\mu_{00} & \mu_{\mathrm{j} 1} \\
\mu_{10} & \mu_{11}
\end{array}\right]\left[\begin{array}{c}
u_{0} \\
-\frac{2}{5 p} q_{0}
\end{array}\right] .
\end{aligned}
$$


The moments of the right side of Eq. (38) can be obtained by using the integral identity given by $\mathrm{Eq}$. (56) as follows. Fi.st, the collisional restoring coefficient terms, after some tedious algebra, lead to

$$
\begin{aligned}
& \int d^{3} v \frac{w^{2}}{3}\left[\begin{array}{c}
L_{0}^{(3 / 2)} \\
L_{1}^{(3 / 2)}
\end{array}\right]\left(\nu_{0} B L_{0}^{(3 / 2)}+\nu_{1} B L_{1}^{(3 / 2)}\right) f_{M} \\
& =\frac{p}{m} \nu_{0} B\left[\begin{array}{l}
1 \\
0
\end{array}\right]+\frac{2}{5 p} g_{\|} B \int d^{3} v \frac{\nu_{\perp}}{v_{T}^{2}} \frac{w^{2}}{3} L_{0}^{(3 / 2)} L_{1}^{(3 / 2)} f_{M}\left[\begin{array}{l}
1 \\
0
\end{array}\right] \\
& =0 .
\end{aligned}
$$

Here, we have used Eqs. (59) and (60) as well as the orthogonality properties of the Laguerre polynomials. Similarly, the remaining terms yield

$$
\begin{aligned}
& \int d^{3} v \frac{w^{2}}{3}\left[\begin{array}{l}
L_{0}^{(3 / 2)} \\
L_{1}^{(3 / 2)}
\end{array}\right]\left(\frac{1}{p} \mathbf{B} \cdot \nabla \cdot \Pi_{\|} L_{0}^{(3 / 2)}+\frac{2}{5 p} \mathrm{~B} \cdot \nabla \cdot \Theta_{\|} L_{1}^{(3 / 2)}\right) f_{M} \\
& =\frac{1}{m}\left[\begin{array}{l}
\mathrm{B} \cdot \nabla \cdot \Pi_{\|} \\
\mathrm{B} \cdot \nabla \cdot \Theta_{\|}
\end{array}\right] .
\end{aligned}
$$

Putting all the these pieces together, we finally obtain from the "proper" moments [Eq. (61)] of Eq. (38)

$$
\left[\begin{array}{l}
3 \cdot \nabla \cdot \Pi_{\|} \\
\mathbf{B} \cdot \nabla \cdot \Theta_{\|}
\end{array}\right]=n m\left\langle B^{2}\right\rangle \hat{f}_{t}(\theta)\left[\begin{array}{ll}
\mu_{00} & \mu_{01} \\
\mu_{10} & \mu_{11}
\end{array}\right]\left[\begin{array}{c}
u_{\theta} \\
-\frac{2}{5 p} q_{\theta}
\end{array}\right]+\mathbf{B} \cdot \nabla\left[\begin{array}{c}
\alpha_{0}(\theta) \\
\alpha_{1}(\theta)
\end{array}\right] \text {. }
$$

The last terms in the above equation describe variations within the magnetic fivx surface that vanish upon averaging over the flux surface. These functions are given by

$$
\left[\begin{array}{c}
\alpha_{0}(\theta) \\
\alpha_{1}(\theta)
\end{array}\right]=\frac{m\left\langle B^{2}\right\rangle^{1 / 2}}{B f_{c}} \int d^{3} v w_{\|} u_{\|} F_{1}\left[\begin{array}{l}
L_{0}^{(3 / 2)} \\
L_{1}^{(3 / 2)}
\end{array}\right]
$$

Averaging over the poloidal flux surface, we easily recover the previous result $^{1,4}$ for the flux-surface-averaged viscous and heat viscous forces, namely

$$
\left[\begin{array}{c}
\left\langle\mathbf{B} \cdot \nabla \cdot \Pi_{\|}\right\rangle \\
\left\langle\mathbf{B} \cdot \nabla \cdot \Theta_{\|}\right\rangle
\end{array}\right]=n m\left\langle B^{2}\right\rangle\left[\begin{array}{ll}
\mu_{00} & \mu_{01} \\
\mu_{10} & \mu_{11}
\end{array}\right]\left[\begin{array}{c}
u_{0} \\
-\frac{2}{5 p} q_{0}
\end{array}\right] \text {. }
$$


Since the spatial shape factor $\hat{f}_{t}(\theta)$ is not expressible in terms of elementary functions, it is plotted in Figure 1. The poloidal angle variation of the viscous stress in Pfirsch-Schlüter and plateau collisionality regimes ${ }^{8,9}(\propto$ $\sin ^{2} \theta$ ) is plotted in Figure 2 for comparison. Here, all of the poloidal angle dependence factors are normalized to yield unity upon averaging. A typical tokamak magnetic field is plotted beneath each of the above two figures for convenient reference. As we can see from the figures, the poloidal angle dependence factor $\hat{f}_{t}(\theta)$ in the banana regime is mostly a cosine-like function of the poloida! angle $\theta[\sim \cos (\theta / 2)]$, which has its maximum localized on the outside of the the toramak (at $\theta=0$ ) and its minimum on the inside $(\theta= \pm \pi)$.

The spatial shape factor in the Pfirsch-Schlüter and plateau regime is proportional to $(\mathbf{b} \cdot \nabla \ln B)^{2} \sim \sin ^{2} \theta$ - because the collisional magnetic pumping is proportional to the local parallel gradient in the magnetic field strength. ${ }^{8,0}$ In the banana regime the dominant physical effect is characterized by the viscous drag on the untrapped particle poloidal flow and heat flows, which is caused primarily by the trapped particles that are located on the outer side of the torus $(\theta \sim 0)$. Thus, as shown in Fig. 1, its poloidal dependence is approximately the same as the trapped particle density distribution $\hat{n}_{t}(\theta)$ - see Eq. (77) below. In the Pfirsch-Schlüter and plateau regime on the other hand, the magnetic pumping driving the $P_{2}$-type stress distortions of the distribution function has its maximum at the bottom and top of the tokamak.

In order to determine the details of those B. $\nabla(\cdots)$ terms, it would apparently be necessary to determine an explicit solution for $F_{1}$. This solution must have a discontinuity at the trapped-untrapped boundary, as can be proved by integrating Eq. (38) over a boundary layer from $\lambda_{c}-0$ to $\lambda_{c}+0$ while making use of Eqs. (41) and (54), because there exists a discontinuity in the slope of $F_{0}$ at this boundary (see Fig. 3 ). Nevertheless, the calculation in this paper of the variation of average part of the parallel viscous stress forces within the flux surface that has been achieved through the new ChapmanEnskog-like approach is certainly a step forward and should be quite useful 
in numerical simulations of neoclassical MHD instabilities, turbulence and transport. ${ }^{10,11}$

Another application of the poloidal angle variation result is that it can be used to determine the density and potential poloidal variation within a magnetic flux surface. For example, adding the electron and ion momentum balance equations and assuming no temperature gradient, we find that in the banana collisionality regime

$$
\begin{aligned}
\left(T_{e}\right. & \left.+T_{i}\right)(\mathbf{B} \cdot \nabla) ; n_{1}(\psi, \theta) \\
& \simeq\left\langle\mathbf{B} \cdot \nabla \cdot\left(\Pi_{e}+\Pi_{\mathbf{i}}\right)\right\rangle-\mathbf{B} \cdot \nabla \cdot\left(\Pi_{\mathrm{e}}+\Pi_{\mathbf{i}}\right) \\
& \propto \frac{\dot{\mu}}{\Omega_{p}} \cos \frac{\theta}{2}
\end{aligned}
$$

which yields

$$
\frac{n_{1}}{n_{0}} \propto \frac{\mu}{\Omega_{p}} \sin \frac{\theta}{2}
$$

where $\mu$ is $\mu_{00}$ and $\Omega_{p}=e B_{p} / m c$ is the poloidal gyro-frequency. The density variation within a flux surface is usually very small since the ratio of the viscosity to poloidal gyro frequency is typically $10^{-4}$ or smaller.

From the preceding direct derivation, the somewhat complicated mathematical computations can yield a simple physical interpretation for the parallel viscous stresses, as follows. First, note that the parallel viscous forces are determined by velocity-space moments of

$$
u_{\|} \frac{\nu_{\perp}}{2} £\left(w_{\|} B-\frac{\left\langle B^{2}\right\rangle^{1 / 2}}{f_{c}} u_{\|}\right) \frac{m}{T}\left(u_{0} L_{0}^{(3 / 2)}-\frac{2}{5 p} q_{0} L_{1}^{(3 / 2)}\right) f_{M} .
$$

Second, note that the $w_{\|} B$ term represents the parallel free streaming while the $\frac{\left\langle B^{2}\right\rangle^{1 / 2}}{f_{c}} u_{\|}$term (see Fig. 3) represents the parallel flow of the untrapped particles. Thus, $w_{\|} B-\frac{\left\langle B^{2}\right\rangle^{1 / 2}}{f_{c}} u_{\|}$, which is plotted in Fig. 3, can be interpreted as the "collisionless" or stress-inducing $\left[P_{2}\right.$-dependence induced by collisions via Eq. (38)] variation in the distribution function caused by the untrapped particle's bouncing motion over the local magnetic mirrors since it can be checked that $F_{0}$ has null $\left(w_{\|} L_{0}^{(3 / 2)}, w_{\|} L_{1}^{(3 / 2)}\right)$ and $\left(L_{0}^{(1 / 2)}, L_{1}^{(1 / 2)}\right)$ moments. 
Third, note that the collisional scattering acting upon $w_{\|} B-\frac{\left\langle B^{2}\right\rangle^{1 / 2}}{f_{c}} u_{\|}$produces the collisional "viscous drag" forces on the flows in the poloidal $(\nabla B$ within the flux surface) direction in a tokamak that have been calculated above. Firally, note that the above moment characterizes the collisions of the mostly free streaming untrapped particles with the "stationary" trapped particles and to a lesser extent with the barely untrapped particles whose parallel motion is modulated by their motion over the magnetic mirrors, both of which are proportional to $\sqrt{\epsilon}$. These are the physical reasons why the parallel viscous stress forces are proportional to $\sqrt{\epsilon} \nu$ and the poloidal flow components.

\section{DISCUSSION AND CONCLUSION}

To clarify the various kinds of approximations involved in the derivation of the flux surface averaged parallel viscous stresses it is necessary to give a summary and brief discussion of the analytical computation of $\left\langle\mathbf{B} \cdot \nabla \cdot \Pi_{\|}\right\rangle$and $\left\langle\mathrm{B} \cdot \nabla \cdot \Theta_{\|}\right\rangle$. The starting point used in this paper is the Chapman-Enskoglike version of the drift kinetic equation which is valid to first order in the small gyro-radius approximation [see Eq. (2)]. A slow transport time scale is assumed to neglect the temporal dependence of the kinetic distortion $F$ in the drift kinetic equation and also to justify the conventional incompressibility approximations, ${ }^{1} \nabla \cdot \mathbf{V}=0$ and $\nabla \cdot \mathbf{q}=0$, in neoclassical theory. To solve the equation for the kinetic distortion $F$ in the banana collisionality regime, a subsidiary ordering scheme has been utilized assuming large untrapped particle bouncing frequency relative to the typical collision frequency, i.e. $\nu \ll \epsilon^{3 / 2} \omega_{b}$. We would also expect ${ }^{1}$ small corrections of higher order of $[l(l+1)]^{-1}$, where $l$ is order in the Legendre polynomial expansion, to the solution of the lowest order kinetic distortion $F_{0}$, due to terms neglected in the approximate collision operator given in Eq. (4). Within the two Laguerre polynomial ( $L_{0}, L_{1}$ or two by two moment) approach, the $L_{j}$ (with $j \geq 2$ ) expansions are unimportant because the distribution function is reasonably smooth in its energy dependence and only the $L_{0}^{(l+1 / 2)}$ and 
$L_{1}^{(l+1 / 2)}$ moments are relevant in the $n, T, \mathrm{~V}, \mathrm{q}$ fluid moment approximations. In fact, the corrections caused by these higher order moments are of the order of a few percent, which is about the inherent inaccuracy $1 / \ln \Lambda_{\alpha \beta}$ in the Fokker-Planck Coulomb collision operator which ignores large angle scattering. ${ }^{12}$ Hirshman and Sigmar ${ }^{1}$ have made the argument that a highly structured energy dependence for the the kinetic distortion $F$ is not required because of the variational nature of the transport coefficients. ${ }^{13,14}$

The direct calculation of the the neoclassical viscous forces presented in this paper has shown that it is the presence of the $P_{1}\left(w_{\|} / w\right)$-type driving terms that are needed fo: momentum conservation in the kinetic equation. They are what led to the direct derivation of the viscous stress forces. In contrast, the previous derivations ${ }^{1,2,6}$ have had to use a subtle procedure to effectively cancel various terms to reinstall the momentum conserving properties in the resultant equations and thereby infer the neoclassical viscous forces. Application of this formalism to the problem of calculating the neoclassical viscous stress forces has demonstrated the advantages and directness of this hybrid fluid/kinetic moment approach. Through this new ChapmanEnskog-like approach, we have been able to determine, for the first time, the poloidal variation of the parallel viscous forces $B \cdot \nabla \cdot \Pi_{\|}$and $B \cdot \nabla \cdot \Theta_{\|}$in the banana collisionality regime. It has been shown that the variations of the parallel viscous forces are governed by spatial shape factor $\hat{f}_{\ell}(\theta)$ in the banana regime and that this factor is substantially different from that in the PfirschSchlüi ar and plateau regimes. The former is approximately $\cos (\theta / 2)$ with its maximuin located at the outside $(\theta=0)$ of the tokamak confinement region where particles are trapped and its minimum located inside $(\theta= \pm \pi)$. The approximate $\cos (\theta / 2)$ dependence is also physically reasonable considering that the fraction of the trapped particles is

$$
\frac{n_{t}}{n} \equiv \frac{\int_{t} d^{3} v f}{\int d^{3} v f} \simeq \sqrt{2 \epsilon} \cos (\theta / 2) .
$$

In the more collisional plateau and Pfirsch-Schlüter regimes the parallel viscous forces is proportional to $(b \cdot \nabla \ln B)^{2} \sim \sin ^{2} \theta$ and reflects the collisional 
magnetic pumping effects which are maximum at the top and bottom of the torus $(\theta= \pm \pi / 2)$ and minimum at the insice $(\theta=\pi)$ and outside $(\theta=0)$ where $\mathbf{b} \cdot \nabla B=0$. Therefore, we can conclude that the radial neoclassical transport in the banana regime is mainly localized on the outside of a tokamak, while in more collisioral (Pfirsch-Schlüter and plateau) regimes it is localized at the top and botiom of the tokamak.

\section{ACKNOWLEDGMENTS}

Beneficial discussions with Drs. D.J. Sigmar and C.T. Hsu are gratefully acknowledged. This research was supported by U.S. Department of Energy Grant No. DE-FG02-86ER53218. 


\section{Appendix A. VISCOSITY COEFFICIENTS}

The viscosity coefficients in the banana collisionality regime are the same as those given by Hirshman and Sigmar [3] previously:

$$
\begin{aligned}
& \mu_{00}=\frac{f_{t}}{f_{c}} \frac{8}{3 \sqrt{\pi}} \int_{0}^{\infty} \frac{v^{4} d v}{v_{T}^{5}} \frac{\nu_{\perp}}{2} \exp \left(-v^{2} / v_{T}^{2}\right)\left[L_{0}^{(3 / 2)}\right]^{2} \\
& \mu_{01}=\frac{f_{t}}{f_{c}} \frac{8}{3 \sqrt{\pi}} \int_{0}^{\infty} \frac{v^{4} d v}{v_{T}^{5}} \frac{\nu_{\perp}}{2} \exp \left(-v^{2} / v_{T}^{2}\right) L_{0}^{(3 / 2)} L_{1}^{(3 / 2)} \\
& \mu_{10}=\mu_{01} \\
& \mu_{11}=\frac{f_{t}}{f_{c}} \frac{8}{3 \sqrt{\pi}} \int_{0}^{\infty} \frac{v^{4} d v}{v_{T}^{5}} \frac{\nu_{\perp}}{2} \exp \left(-v^{2} / v_{T}^{2}\right)\left[L_{1}^{(3 / 2)}\right]^{2}
\end{aligned}
$$

For electrons and ions, these become

$$
\begin{aligned}
\mu_{00}^{e} & =\frac{f_{t}}{f_{c} \tau_{e e}}\{Z+\sqrt{2}-\ln (1+\sqrt{2})\} \stackrel{Z \equiv 1}{=} 1.533 \frac{f_{l}}{f_{c} \tau_{e e}} \\
\mu_{01}^{e} & =\frac{f_{t}}{f_{c} \tau_{e e}}\left\{\frac{3}{2} Z+\frac{4}{\sqrt{2}}-\frac{5}{2} \ln (1+\sqrt{2})\right\} \stackrel{Z \equiv 1}{=} 2.125 \frac{f_{t}}{f_{c} \tau_{e e}} \\
\mu_{11}^{e} & =\frac{f_{t}}{f_{c} \tau_{e e}}\left\{\frac{13}{4} Z+\frac{39}{4 \sqrt{2}}-\frac{25}{4} \ln (1+\sqrt{2})\right\} \stackrel{Z \equiv 1}{=} 4.636 \frac{f_{l}}{f_{c} \tau_{c c}} \\
\mu_{00}^{i} & =\frac{f_{t}}{f_{c} \tau_{i i}}\{\sqrt{2}-\ln (1+\sqrt{2})\}=0.533 \frac{f_{t}}{f_{c} \tau_{i i}} \\
\mu_{01}^{i} & =\frac{f_{t}}{f_{c} \tau_{i i}}\left\{\frac{4}{\sqrt{2}}-\frac{5}{2} \ln (1+\sqrt{2})\right\}=0.625 \frac{f_{t}}{f_{c} \tau_{i i}} \\
\mu_{11}^{i} & =\frac{f_{t}}{f_{c} \tau_{i i}}\left\{\frac{39}{4 \sqrt{2}}-\frac{25}{4} \ln (1+\sqrt{2})\right\}=1.386 \frac{f_{t}}{f_{c} \tau_{i i}}
\end{aligned}
$$

In the preceeding the electron-electron collision time is given by

$$
\frac{1}{\tau_{e e}} \equiv \frac{4}{3 \sqrt{\pi}} \frac{4 \pi n_{e} e^{4} \ln \Lambda}{m_{e}^{2} v_{T_{e}}^{3}},
$$

and the ion-ion collision time is given by

$$
\frac{1}{\tau_{i i}} \equiv \frac{4}{3 \sqrt{\pi}} \frac{4 \pi n_{i} Z_{i}^{2} e^{4} \ln \Lambda}{m_{i}^{2} v_{T_{i}}^{3}} .
$$




\section{References}

${ }^{1}$ S. P. Hirshman and D. J. Sigmar, Nucl. Fusion 21, 1079 (1981).

${ }^{2}$ K. C. Shaing and J. D. Callen, Phys. Fluids 26, 1526 (1983).

${ }^{3}$ J. P. Wang and J. D. Callen, Phys. Fluids B 4, 1139 (1992).

${ }^{4}$ J. D. Callen, Z. Chang, and J. P. Wang, "Direct derivation of neoclassical viscosity coefficients in tokamaks", in 17th EPS Conference on Controlled. Fusion and Plasma Heating, Vol. 14B Part II (Amsterdam, .Tune, 1990), p. 852-855. See also Appenulix in Y. B. Kim, P. H. Diamond, H. Biglari and J. D. Callen, Phys. Fluids B 3, 384(1991).

${ }^{5}$ F. L. Hirton and R. D. Hazeltine, Rev. Mod. Phys. 48, 239 (1976).

${ }^{6}$ M. N. Rosenbluth, R. D. Hazeltine, and F. L. Hinton, Phys. Fluids 15, 116 (1972).

${ }^{7}$ S.P. Hirshman and D. J. Sigmar, Phys. Fluids 19, 1532 (1976).

${ }^{8}$ A. B. Hassam and R. M. Kulsrud, Phys. Fluids 21, 2271 (1978).

${ }^{9}$ W. M. Stacey Jr., A. W. Bailey, D. J. Sigmar, and K. C. Shaing, Nucl. Fusion 25, 463 (1985).

${ }^{10}$ J. D. Callen, W.X. Qu, K.D. Siebert, B.A. Carreras, K.C. Shaing, and D.A. Spong, in Plasma Physics and Controlled Nuclear Fusion Research 1986 (IAEA, Vienna, 1987), Vol. II, p.157.

${ }^{11}$ J. D. Callen, Y. B. Kim, A. K. Sundaram, B. A. Carreras, L. Garcia, K. C. Shaing, D. A. Spong, H. Biglari, P. H. Diamond, and O. J. Kwon, in Plasma Physics and Controlled Nuclear Fusion Research 1988, (IAEA, Viena, 1989), Vol. II, p.53.

${ }^{12}$ M. N. Rosenbluth, W. M. MacDonald, and D. L. Judd, Phys. Rev. 107, 1 (1957). 
${ }^{13}$ P.H. Rutherford, Phys. Fluids 13, 482 (1970).

${ }^{14}$ B.B. Robinson and I.B. Bernstein, Ann. Phys. 18, 110 (1962). 


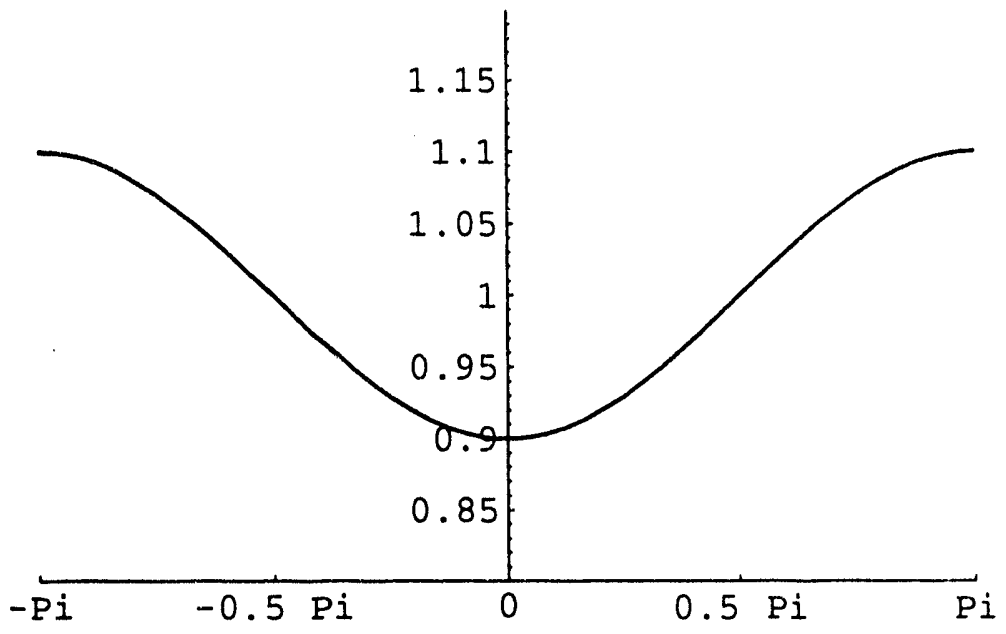

(a)

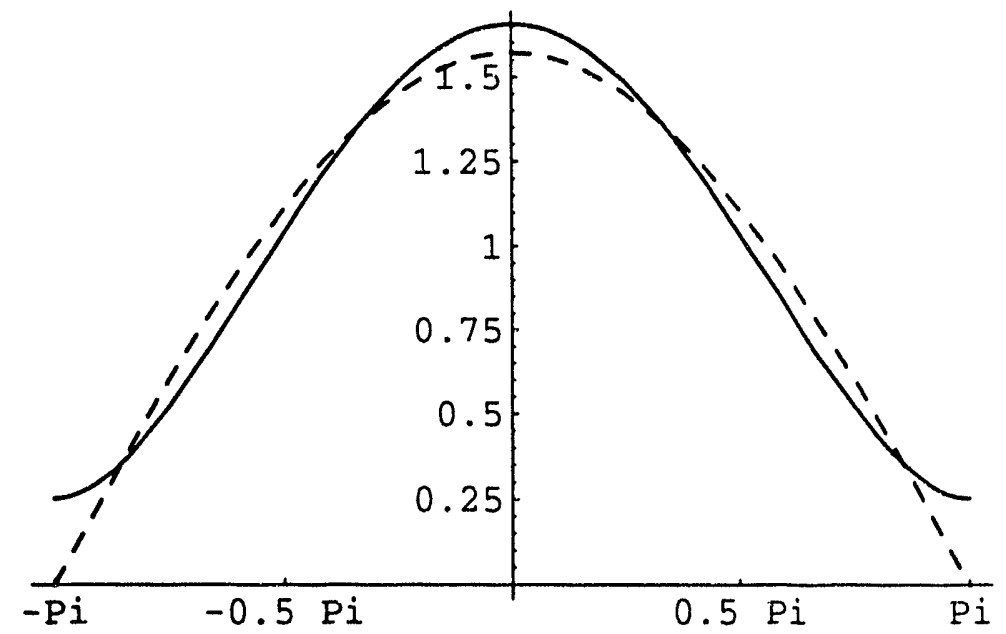

(b)

Figure 1: (a) Tokamak magnetic field versus the poloidal angle $\theta$. (b) Variation of the flow weighted fraction $\hat{f}_{t}$ (solid) and $\hat{n}_{t}(\theta) \equiv \frac{\pi}{2} \cos (\theta / 2)$ (dashed) in the banana regime versus the poloidal angle $\theta$. For both of these plots the inverse aspect ratio $\epsilon=0.1$. 


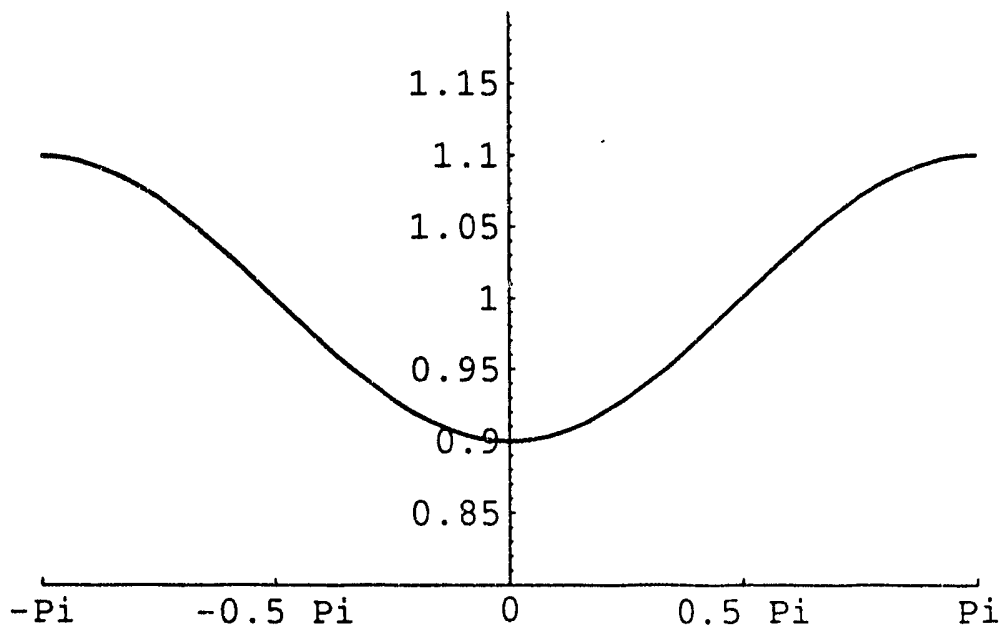

(a)

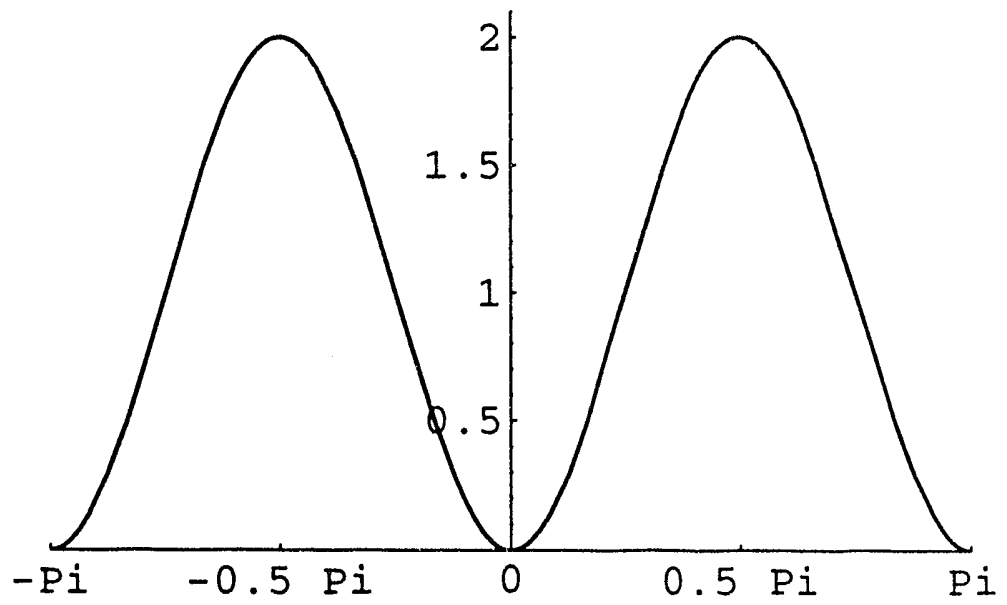

(b)

Figure 2: (a) Tokamak magnetic field versus the poloidal angle $\theta$ with $\epsilon=0.1$. (b) Poloidal angle variation of the parallel viscous force in the Pfirsch-Schliiter and plateau collisionality regimes. 


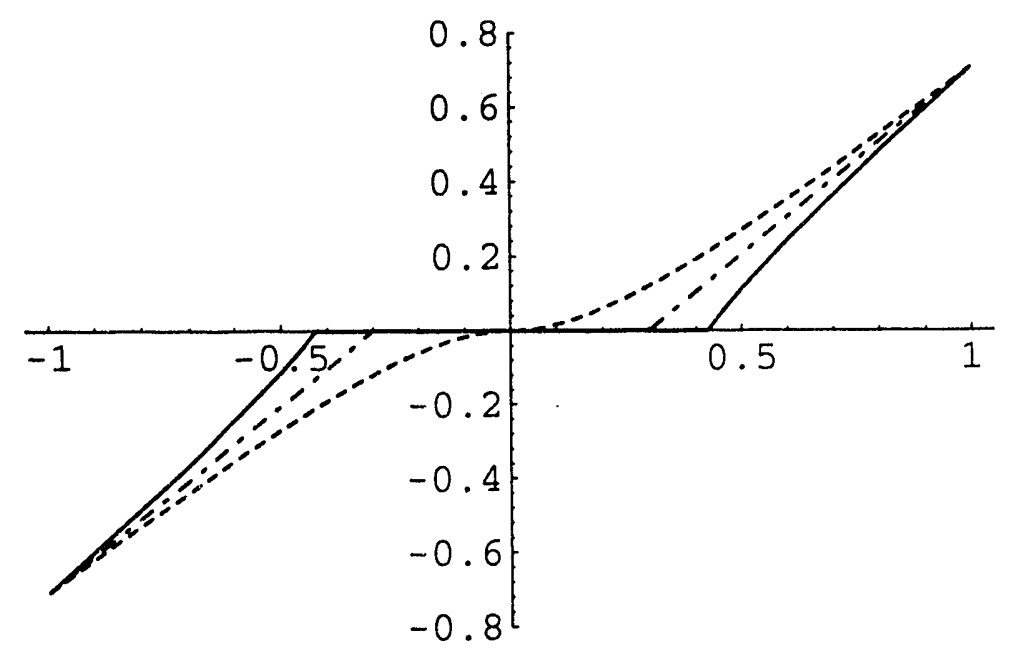

(a)

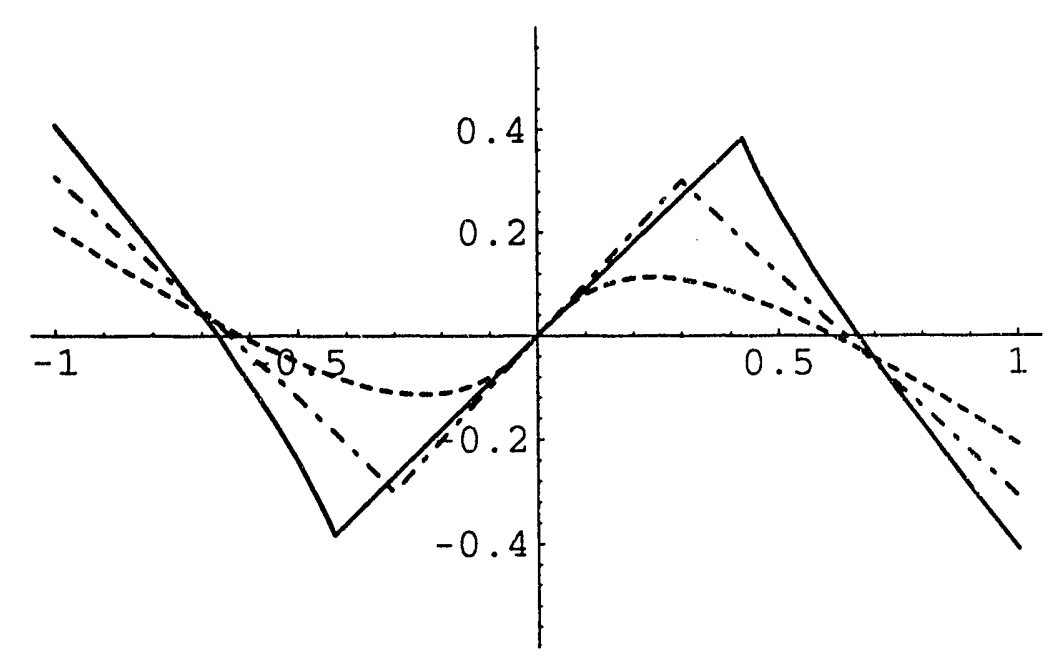

(b)

Figure 3: (a) $u_{\|} / w$ versus $w_{\|} / w$ for given $\theta$ values. (b) $\frac{w_{\|}}{w} \frac{B}{\left\langle B^{2}\right\rangle^{1 / 2}}-\frac{1}{f_{c}} \frac{u_{\|}}{w}$ (proportional to the distribution function $F_{0}$ ) verus $w_{\|} / w$. For both of these plots, the solid curve is plotted with $\theta=0$, the dot-dashed with $\theta=\pi / 2$, and the dashed with $\theta=\pi$. A discontinuity in slope occurs at the trappeduntrapped boundary. 

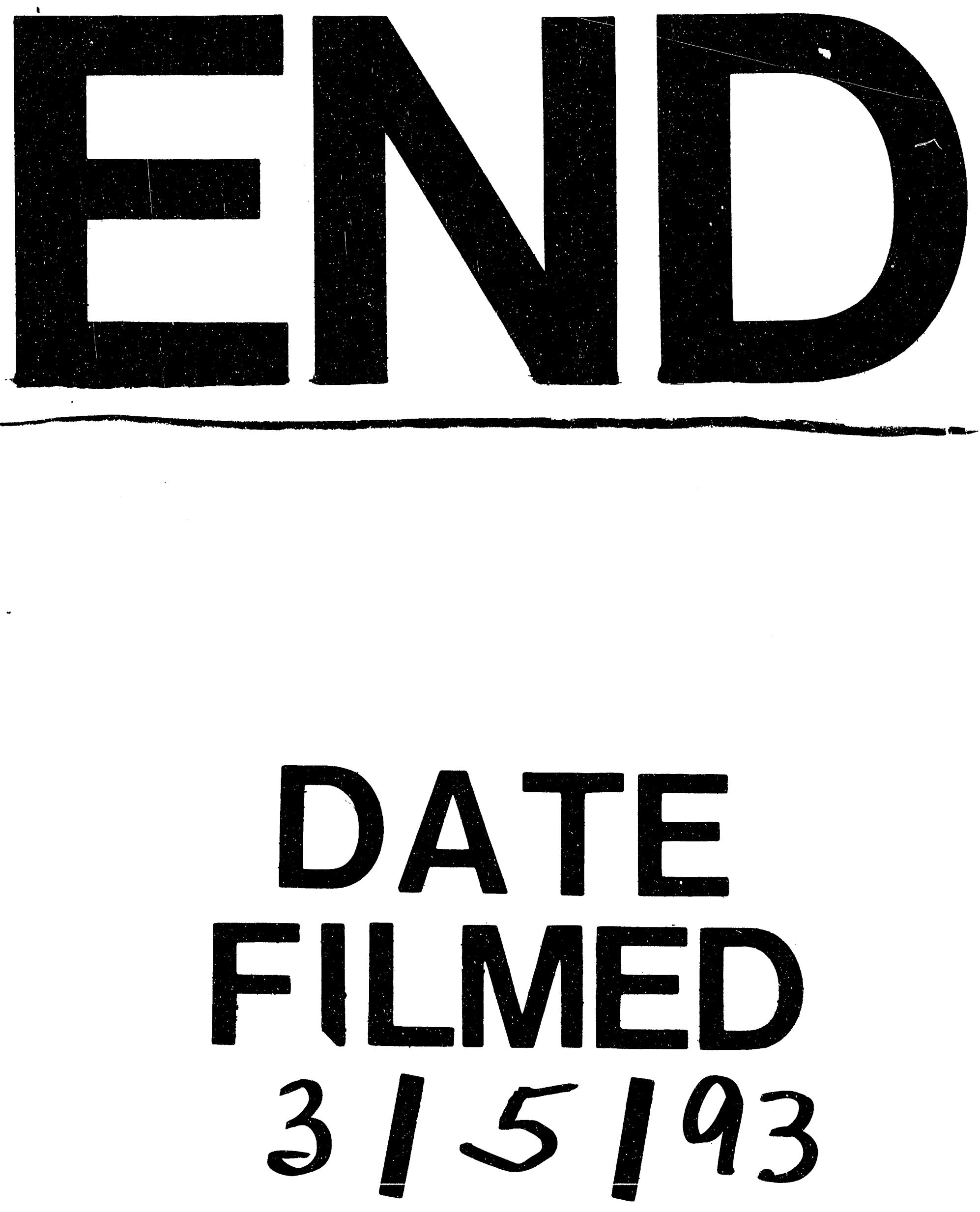
1 